\section{Síndrome de Phelan-McDermid: reporte de un caso y revisión de la literatura}

Hernández-Gómez $\mathrm{M}^{1}$, Meléndez-Hernández $\mathrm{R}^{2}$, Ramírez-Arroyo $\mathrm{E}^{3}$, MayénMolina DG ${ }^{4}$

\begin{abstract}
Resumen
El síndrome de Phelan-McDermid (SPMD) es un desorden del neurodesarrollo, también Ilamado síndrome de deleción 22q13.3, que genera la pérdida de la función del gen SHANK3. Se caracteriza por hipotonía neonatal severa, retardo global del desarrollo, retraso severo o ausencia de lenguaje y dismorfias menores. El $80 \%$ de los casos son de novo y, a pesar de que su prevalencia es desconocida, se han descrito aproximadamente 1200 casos alrededor del mundo. Es una causa frecuente de desorden con espectro autista y de discapacidad intelectual, contribuyendo con el 0.5 a $2 \%$ de todos los casos. El método diagnóstico de elección es el estudio de microarreglos acompañado de citogenética convencional. Este artículo describe un caso de SPMD, diagnosticado hasta los cuatro años, con datos clínicos altamente sugestivos desde temprana edad. Es un caso de novo debido a una deleción de $4.3 \mathrm{Mb}$, resultado de la formación de un anillo cromosómico. Consideramos que mediante la difusión de este caso contribuiremos a la divulgación del SPMD, lo que puede permitir, junto con la tecnología diagnóstica actual, que los pacientes y sus familiares se beneficien de un diagnóstico más rápido, oportuno y de un mejor manejo.
\end{abstract}

PALABRAS CLAVE: deleción 22q13.3, síndrome de Phelan-McDermid, desorden genómico, gen SHANK3.

Acta Pediatr Mex. 2018 Jan;39(1):42-51.

\section{Phelan-McDermid syndrome: A case report and literature review.}

Hernández-Gómez $\mathrm{M}^{1}$, Meléndez-Hernández $\mathrm{R}^{2}$, Ramírez-Arroyo $\mathrm{E}^{3}$, MayénMolina DG ${ }^{4}$

\footnotetext{
Abstract

Phelan-McDermid syndrome is a neurodevelopmental disorder, also called 22q13.3 deletion syndrome, resulting in the loss of function of the gene SHANK3. It is characterized by severe neonatal hypotonia, global developmental delay, sever speech delays or absence of language and minor dysmorphic features. $80 \%$ of cases are de novo, although their prevalence is unknown, approximately 1200 cases have been described around the world. It is a frequent cause of autism spectrum disorder and intellectual disability, contributing 0.5 to $2 \%$ of all cases. Microarray is the study of choice with conventional citogenetics. In this report we present one case of Phelan-McDermid
}

${ }^{1}$ Médico genetista, Especialista en Genética perinatal. Biólogo del Laboratorio de Citogenética.

${ }^{3}$ Química del Laboratorio de Genética Molecular. ${ }^{4}$ Médico-Jefe de la Unidad de Genética.

Unidad de Genética Aplicada del Hospital Ángeles Lomas.

Recibido: 22 de junio del 2016

Aceptado: 21 de marzo del 2017

Correspondencia

Mariana Hernández-Gómez

mariana.hernandezgomez@yahoo.com.mx

Este artículo debe citarse como

Hernández-Gómez $M$, Meléndez-Hernández $R$, Ramírez-Arroyo E, Mayén-Molina DG. Síndrome de Phelan-McDermid: reporte de un caso y revisión de la literatura. Acta Pediatr Mex. 2018;39(1):42-51.

DOI: http://dx.doi.org/10.18233/APM1No1pp42-511539 
syndrome, with highly suggestive clinical data at a very early age who was diagnosed until 4 years of age. It is a de novo case, due to a deletion of $4.3 \mathrm{Mb}$ resulting from the formation of a chromosomal ring. We believe that by disseminating this case, we will contribute to the disclosure of this entity, which may allow, together with the current diagnostic technology, that patients and their families benefit from a faster, timely diagnosis and a better follow-up.

KEYWORDS: 22q13.3 deletion; Phelan-Mcdermid syndrome; genomic disorder; SHANK3 gene

\section{Correspondence}

Mariana Hernández-Gómez

mariana.hernandezgomez@yahoo.com.mx

\section{INTRODUCCIÓN}

El síndrome de Phelan-McDermid (SPMD; OMIM ID 606232) o deleción 22q13.3 fue descrito por primera vez en 1985 . Es un desorden del neurodesarrollo caracterizado por hipotonía neonatal severa, retardo global del desarrollo, ausencia o retraso severo del lenguaje, dismorfias menores y espectro autista. Se han documentado alrededor de 1200 casos en todo el mundo, pero su frecuencia no se conoce con exactitud debido a la dificultad en el diagnóstico. A pesar de esto, se considera la segunda microdeleción en frecuencia, después de la deleción 1p36, y es una causa importante de autismo y discapacidad intelectual contribuyendo entre 0.5 y $2 \%$ de todos los casos. Ocurre en igual proporción en hombres que en mujeres. ${ }^{1-4}$

La pérdida de la región 22q13.3 puede ser resultado de una deleción terminal, de una translocación o de la formación de un anillo del brazo largo del cromosoma 22, alteraciones que pueden ser de novo o heredadas. Dentro de esta región se localizan más de 90 genes pero la región crítica del SPMD se ha ido limitando a un área aproximadamente de $100 \mathrm{~kb}$ que contiene tres genes: $A C R$, SHANK3 y RABL2B, de los cuales $S H A N K 3$ es el gen vinculado con la mayor parte del fenotipo observado en el SPMD. ${ }^{4}$ SHANK3 se encuentra expresado en diferentes áreas del cerebro y codifica para una proteína que se localiza en la densidad postsináptica conectando a los receptores de membrana con la actina del citoesqueleto, brindando estabilidad e integridad a la estructura sináptica. ${ }^{2,4}$

El diagnóstico se realiza mediante microarreglos, estudios de citogenética convencional y molecular y otras técnicas de biología molecular en las que se demuestre la pérdida de la región 22q13.3 o del gen SHANK3. ${ }^{1}$

Presentamos el caso de un niño con SPMD y la revisión de la literatura.

\section{CASO CLÍNICO}

Niño de 6 años de edad, producto de la primera gesta, sin antecedentes familiares de importancia. Embarazo normoevolutivo, obtenido de término, peso $4 \mathrm{~kg}$ (pc 50), talla $51.5 \mathrm{~cm}$ (pc 50), Apgar 9-9. A los dos meses de edad se diagnosticó reflujo gastroesofágico e inició con episodios de otitis media e infecciones de vías aéreas de repetición. A los 4 meses de edad no tenía interés por el medio, presentaba hipotonía y retraso psicomotor, el balbuceo que había adquirido lo perdió y no volvió a emitir sonidos. Al año de edad se diagnosticó con hipotonía central y retraso psicomotor, se realizó una resonancia magnética cerebral que mostró hipoplasia del cuerpo calloso y aumento del espacio subaracnoideo con atrofia cortical. 
A los 4 años se diagnosticó, por parte de un neurólogo pediatra, con espectro autista por la presencia de alteraciones en la comunicación, conducta y socialización. Mediante rehabilitación logró gateo a los 10 meses, bipedestación y marcha al año 7 meses de edad, a los seis años no corría ni brincaba sobre los dos pies, sólo pronunciaba dos bisílabos y no tenía control de esfínteres. Se continuó con terapia motora, de lenguaje y conductual con lo que se logró que realizara tareas de la vida cotidiana. Tiene movimientos estereotipados, como meterse constantemente la mano a la boca, bruxismo y ligera agresividad, así como alteración del ciclo sueño-vigilia y en la percepción del dolor. A la exploración física: peso $22 \mathrm{~kg}$ (pc 50), talla $115 \mathrm{~cm}$ (pc 50), con leve tendencia a la braquicefalia, frente regular, cejas arqueadas, fisuras palpebrales rectas, pestañas largas y abundantes, nariz con punta bulbosa, labios y paladar íntegros, dientes con datos de bruxismo, pabellones auriculares prominentes y acopados. Resto de la exploración sin datos relevantes (Figura 1).

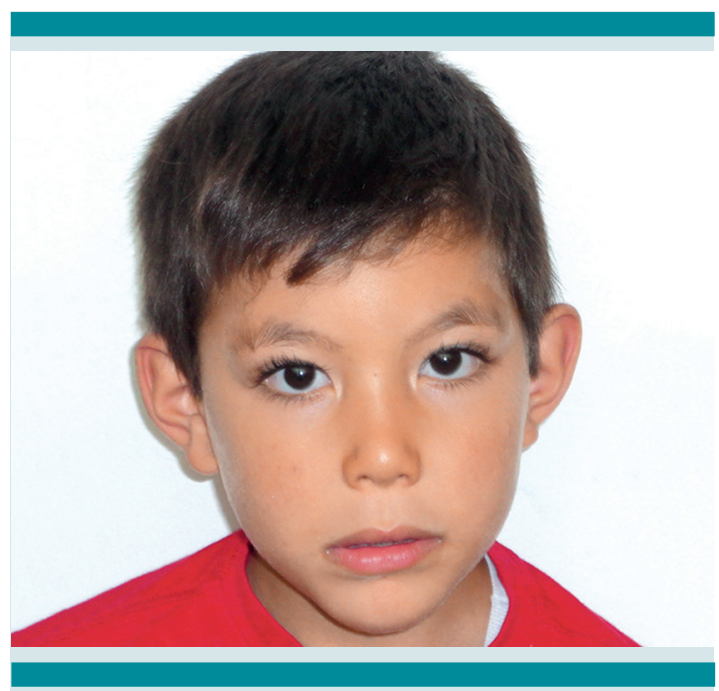

Figura 1. Dismorfias faciales: pestañas abundantes y largas, nariz bulbosa y orejas prominentes.

Nota: los autores solicitamos y obtuvimos consentimiento informado, por ambos padres, para la publicación de este caso y la fotografía tal cual se presenta; mismo que hemos hecho llegar al comité editorial de la revista en cumplimiento con los estándares de publicación.
Estudios realizados por Genética:

- Cariotipo en sangre periférica con bandas GTG que mostró una fórmula cromosómica: 46,XY,r(22)(p12q13) (Figura 2).

- Estudio de hibridación in situ con fluorescencia (FISH por sus siglas en inglés): 46,XY,r(22)(p11.2q13.3)[25].ish r(22) (ARSA-1) (Figura 3).

- Microarreglos con hibridación genómica comparativa (CGH CYTOSCAN 750K): arr

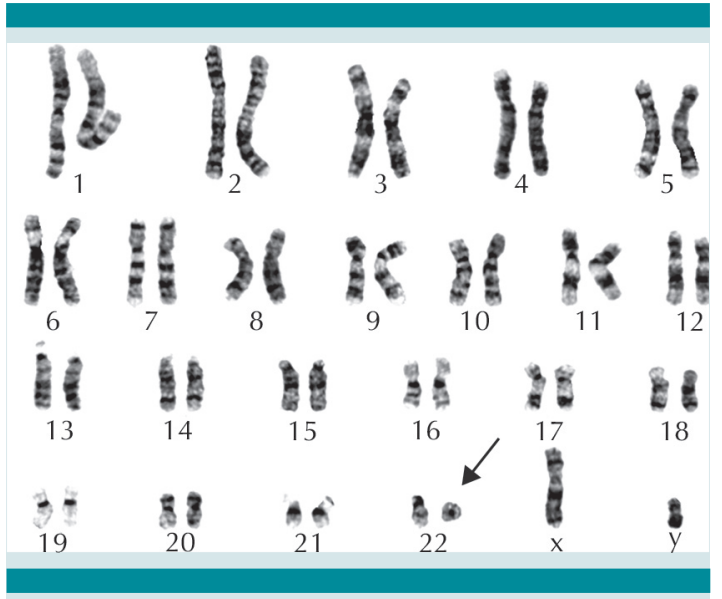

Figura 2. Cariotipo en sangre periférica: $46, X Y, r(22)$ (p12q13).

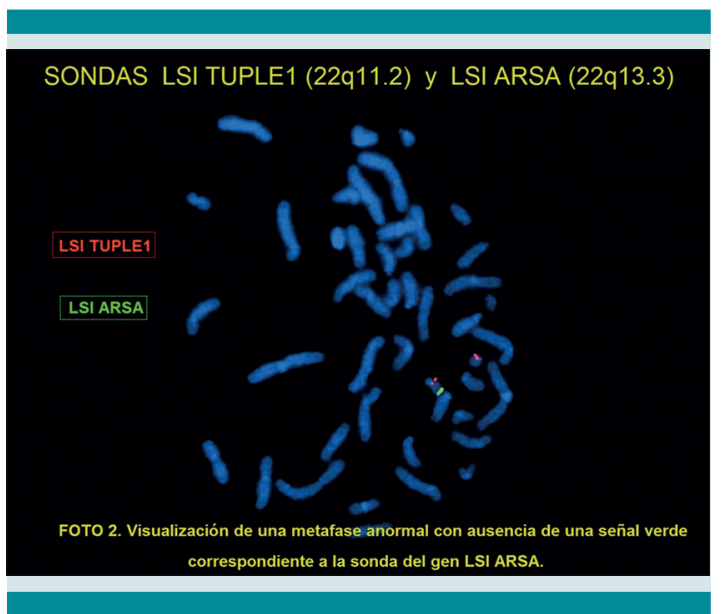

Figura 3. FISH: 46,XY,r(22)(p11.2q13.3) [25]. ish r(22) (ARSA-). 
22q13.31q13.33(46863072-51197766) x1, deleción de $4.3 \mathrm{Mb}$ en 22q13.1q13.33 con deleción de 43 genes enlistados en OMIM

- MLPA (por sus siglas en inglés Multiplex Ligation-Dependent Prube Amplification) 46,XY,r(22) (p11.2q13.3). mlpa22q13.33(P343)x1, que confirma la deleción (Figura 4).

Se realizó cariotipo en ambos padres para descartar alguna alteración estructural cromosómica; en ambos fue normal.

\section{REVISIÓN DE LA LITERATURA}

El SPMD es un síndrome sub-diagnosticado en gran medida porque los datos clínicos no son específicos y en la práctica clínica no se han incluido los microarreglos, como estudio de primera línea, para pacientes con retraso psicomotor con o sin dismorfias, a pesar de que en el año 2010 se estableciera en las guías de práctica clínica. ${ }^{5}$ Por lo que hasta el $30 \%$ de los pacientes con SPMD requieren más de dos estudios de citogenética o biología molecular., ${ }^{4,5}$

A pesar de que el SPMD tiene una gran heterogeneidad clínica se puede decir que la presentación común incluye: retardo del desarrollo y lenguaje, dismorfias faciales, hipotonía y espectro autista, así como alteraciones en el comportamiento que incluyen conductas repetitivas, movimientos estereotipados, alteraciones en el ciclo sueño/vigilia y reacciones negativas a los cambios de rutina. ${ }^{4}$

\section{Manifestaciones clínicas}

\section{Neurológicas}

La pérdida de la función del gen SHANK3 afecta múltiples sistemas, pero como podemos observar el más afectado es el sistema nervioso. El retraso

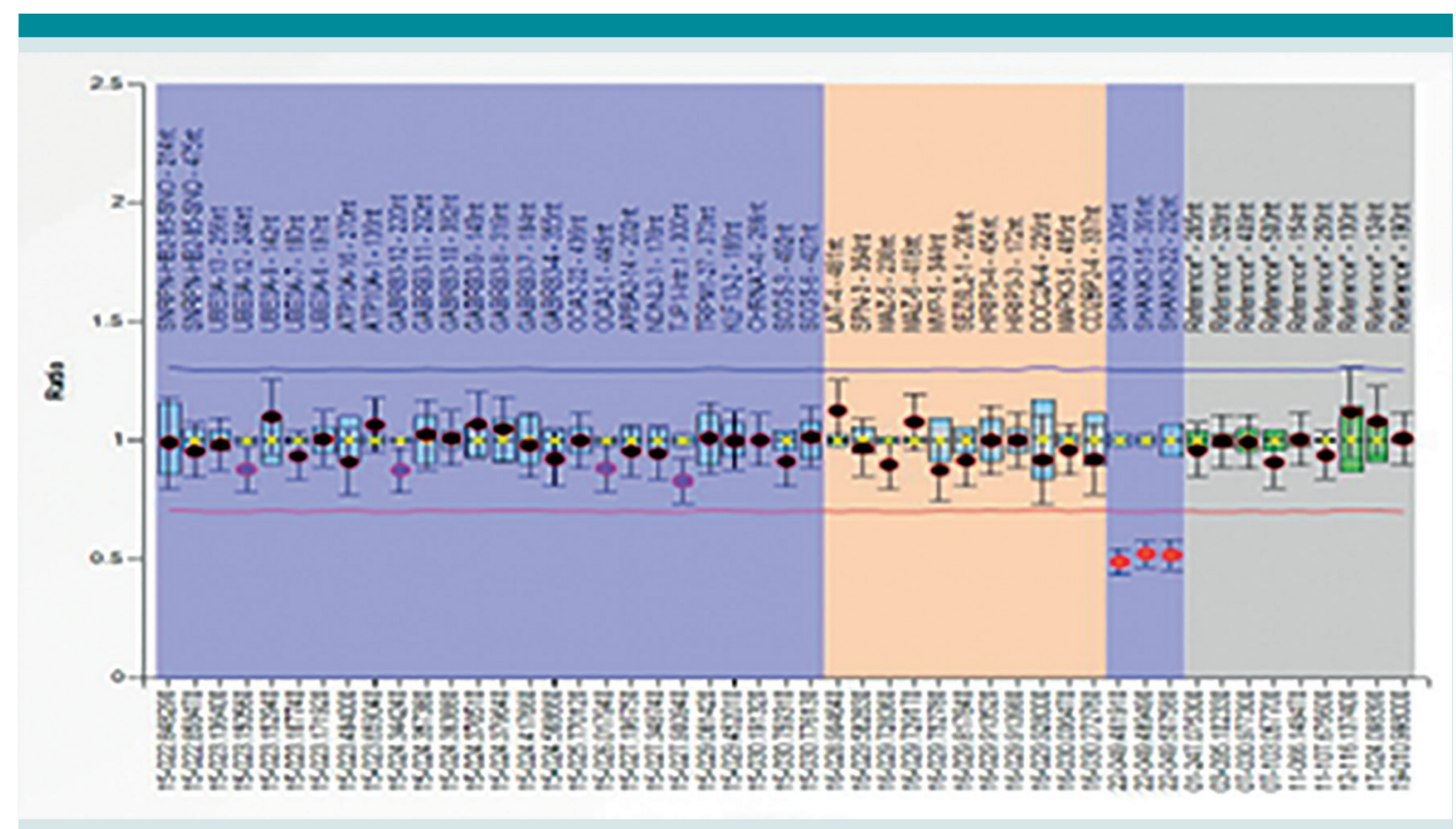

Figura 4. MLPA: 46,XY,r(22)(p11.2q13.3). mlpa 22q13.33(P343)x1 
en el desarrollo puede no hacerse evidente durante los primeros 12 meses de vida, pero uno de los datos clínicos más importantes al nacimiento es la hipotonía, que en nuestro paciente fue diagnosticada durante el primer año de vida. El bajo tono muscular y la falta de coordinación contribuye al retraso en la adquisición de habilidades motoras como el gateo y la marcha y esto puede persistir hasta la edad adulta. ${ }^{2} \mathrm{El}$ nivel de afectación de cada paciente puede variar, pero generalmente todos tiene alteraciones en la marcha e incluso hay reportes de algunos sin deambulación. ${ }^{4}$ Se describe que $88 \%$ de los pacientes camina de forma independiente antes de los 3 años (media de 22 meses). ${ }^{6}$ En el caso de nuestro paciente logró la marcha independiente.

El retraso en el lenguaje está presente en más del $75 \%$ de los casos, afectándose más el lenguaje expresivo que el receptivo. En general, tienen muy poca comunicación y sólo logran seguir órdenes simples. La mayoría de los pacientes balbucea a la edad esperada y posee un pobre vocabulario entre los 3 y 4 años de edad, a esta edad pierden la capacidad de hablar de forma permanente. , $, 6,7$ En una serie de 201 pacientes se vio que el $100 \%$ tenían retraso en el lenguaje y de éstos, el 50\% pierde el lenguaje por completo; $27 \%$ tiene un vocabulario menor a 40 palabras; $10 \%$ tiene un lenguaje con más de 50 palabras y $13 \%$ tiene un vocabulario funcional. ${ }^{6}$

El 40\% de los pacientes con SPMD padece crisis convulsivas, suelen manifestarse durante la pubertad, aunque pueden iniciar a menor edad. 1,4,8 $^{1,8}$ La prevalencia de crisis convulsivas incrementa con la edad de $11 \%$ en aquellos pacientes menores de 5 años a $60 \%$ en adultos. ${ }^{6}$ En nuestro paciente aún no se refieren, lo que puede explicarse por la edad, pero es importante mantenerlo en seguimiento electroencefalográfico.

El comportamiento autista o autismo se observa en 19\% de los pacientes entre los 3 y los 4.9 años de edad, mientras que en los pacientes mayores de 18 años en el $60 \%{ }^{6}$
Se recomienda realizar una resonancia magnética de cerebro, ya que alrededor de $75 \%$ de los casos tiene alteraciones neurológicas estructurales como quistes aracnoideos, dilatación ventricular, hipoplasia del cuerpo calloso y alteraciones en la mielinización. ${ }^{4}$ En este caso, se observó hipoplasia del cuerpo calloso y atrofia cortical.

Se observan alteraciones del sueño, percepción alterada del dolor (suele incrementar de 69 a 89\% entre el grupo de pacientes jóvenes y adultos) y alteraciones del comportamiento como: pobre contacto visual, movimientos estereotipados y otras características que suelen Ilamar la atención como bruxismo, agresividad y "pica" , 1,2,4,7, dos de las cuales también presentó nuestro paciente y se describe que algunas tienden a ir disminuyendo con la edad. ${ }^{6}$

\section{Otras alteraciones}

El sistema gastrointestinal está frecuentemente involucrado en el SPMD; incluye reflujo gastroesofágico, constipación, diarrea y vómito cíclico. Los episodios de este último tienden a recurrir durante algunos meses; se presenta hasta en el $25 \%$ de los casos y pueden acompañarse de cefalea, letargia y deshidratación..$^{1,2,4,6-8}$ Existe una prevalencia alta de malformaciones cardiacas $(25 \%)$, las más frecuentes son comunicación interauricular, insuficiencia tricuspídea, ducto arterioso y retorno pulmonar anómalo. ${ }^{4}$ También se observan anormalidades genitourinarias en $40 \%$ de los casos que incluyen: hidronefrosis, agenesia renal y pielectasia, así como displasia renal y riñones multiquísticos en menor frecuencia. No hay estudios que puntualicen las alteraciones endocrinológicas en el SPMD, pero se han descrito casos de hipotiroidismo, diabetes insípida, pubertad precoz (más frecuente en mujeres; menores de 5 años 19\%, entre 5-9 años 50\%) y retardada. ${ }^{1,6}$ Presentan inmunodeficiencias caracterizadas por infecciones de vías aéreas de 
repetición, alergias y asma, dermatitis, celulitis y hepatitis autoinmune. Este paciente presentó reflujo gastroesofágico y durante un periodo importante padeció infecciones de vías aéreas superiores, otitis media de repetición e incluso ameritó adenoidectomia bilateral, el resto de las manifestaciones no se han presentado aún, pero habrá que mantener una estrecha vigilancia ya que muchas de estas manifestaciones son dependientes de la edad. ${ }^{1,2,4,6-8}$

\section{Crecimiento y desarrollo}

La mayoría de los individuos con deleción 22q13.3 están en los límites normales de crecimiento; sin embargo, se describe que hasta el $10 \%$ de los casos tienen talla baja $(<$ percentil $5)$, talla alta ( $>$ percentil 95), microcefalia $(<$ percentil 3) y macrocefalia (> percentil 97). Alguno de los casos con talla baja se ha asociado a la presencia de un anillo cromosómico, lo cual no corresponde a lo observado ya que en este paciente, a pesar de la presencia del anillo, la talla se encuentra en percentil 50. Logran control de esfínteres alrededor de los 6.5 años de edad, en este caso aún no lo tiene. . $^{1,4}$

\section{Dismorfias menores}

Las más comunes son dolicocefalia, pestañas largas, labios prominentes, pabellones auriculares grandes, nariz bulbosa, barba puntiaguda, manos grandes con uñas displásicas o hipoplásicas. En nuestro caso hay tendencia a la braquicefalia y no había alteraciones ungueales..$^{2,8}$ En el Cuadro 1 se muestran los datos clínicos asociados a la deleción 22q13.3 descritos en la literatura y los hallazgos en el paciente.

\section{DIAGNÓSTICO}

El SPDM se caracteriza por un espectro muy amplio de manifestaciones clínicas con expresividad variable y hasta la fecha no se han
Cuadro 1. Manifestaciones clínicas asociadas a la deleción $22 q 13.3$

\begin{tabular}{|c|c|}
\hline$>95 \%$ de los pacientes & Este caso \\
\hline $\begin{array}{l}\text { RPM severo } \\
\text { Retraso severo o ausencia de lenguaje } \\
\text { Hipotonía } \\
\text { Crecimiento normal o acelerado }\end{array}$ & $\begin{array}{l}\text { Sí } \\
\text { Sí } \\
\text { Sí } \\
\text { Normal }\end{array}$ \\
\hline$>75 \%$ de los pacientes & Este caso \\
\hline $\begin{array}{l}\text { Pestañas largas } \\
\text { Orejas prominentes } \\
\text { Manos relativamente grandes } \\
\text { Uñas del pie displásicas } \\
\text { Disminución de la sensibilidad al dolor }\end{array}$ & $\begin{array}{l}\text { Sí } \\
\text { Sí } \\
\text { No } \\
\text { No } \\
\text { Probable }\end{array}$ \\
\hline$>50 \%$ de los pacientes & Este caso \\
\hline $\begin{array}{l}\text { Dolicocefalia } \\
\text { Cejas pobladas } \\
\text { Párpados prominentes } \\
\text { Mejillas prominentes } \\
\text { Ojos de implantación profunda } \\
\text { Ptosis } \\
\text { Intolerancia al calor } \\
\text { Hipoplasia medio facial } \\
\text { Puente nasal ancho } \\
\text { Nariz bulbosa } \\
\text { Hoyuelo presacro }\end{array}$ & $\begin{array}{l}\text { No } \\
\text { Sí } \\
\text { Sí } \\
\text { No } \\
\text { Sí } \\
\text { No } \\
\text { Sí } \\
\text { Sí } \\
\text { Si } \\
\text { Si } \\
\text { No }\end{array}$ \\
\hline Datos observados en > $25 \%$ & Este caso \\
\hline $\begin{array}{l}\text { Vómito cíclico } \\
\text { Estrabismos } \\
\text { Maloclusión dental } \\
\text { Sindactilia de } 2-3 \mathrm{er} \text { dedo del pie } \\
\text { Clinodactilia del } 5^{\circ} \text { dedo } \\
\text { Convulsiones } \\
\text { Linfedema } \\
\text { Reflujo gastroesofágico } \\
\text { Anormalidades renales }\end{array}$ & $\begin{array}{l}\text { No } \\
\text { No } \\
\text { No } \\
\text { No } \\
\text { Sí } \\
\text { No } \\
\text { No } \\
\text { Sí } \\
\text { No }\end{array}$ \\
\hline Menos de $25 \%$ & Este caso \\
\hline $\begin{array}{l}\text { Pérdida de la audición } \\
\text { Quistes aracnoideos } \\
\text { Celulitis }\end{array}$ & $\begin{array}{l}\text { Sí } \\
\text { No } \\
\text { No }\end{array}$ \\
\hline Comportamiento & Este caso \\
\hline $\begin{array}{l}\text { Pobre contacto visual } \\
\text { Movimientos estereotipados } \\
\text { Poca sociabilización } \\
\text { Movimientos de boca } \\
\text { Bruxismo } \\
\text { Agresividad }\end{array}$ & $\begin{array}{l}\text { Sí } \\
\text { Sí } \\
\text { Sí } \\
\text { Sí } \\
\text { Sí } \\
\text { Sí }\end{array}$ \\
\hline
\end{tabular}

Modificado de: Phelan MC. Deletion 22q13. 3 syndrome. Orphanet journal of rare diseases. 2008;3(1):1.

establecidos datos patognomónicos, por lo que el diagnóstico sigue siendo genético. La haploin- 
suficiencia del gen SHANK3 puede evidenciarse mediante distintas metodologías. El cariotipo con una resolución mayor a 500 bandas puede detectar la mayoría de las deleciones 22q13.3; no obstante, se recomienda realizar otros estudios para confirmarla. El estudio FISH es una de estas pruebas y permite descartar una translocación mediante el empleo de una sonda para arilsulfatasa A (ARSA) y con las sondas sub-teloméricas se pueden identificar deleciones distales.

El estudio de MPLA y el análisis con microarreglos permiten identificar desequilibrios como la pérdida de material en la región 22 q13.3 y, en la actualidad, los microarreglos se consideran el "estándar de oro" para el diagnóstico de SPMD. Esto no excluye la realización del cariotipo ya que sólo mediante este estudio se puede evaluar la presencia de re-arreglos estructurales asociados a la deleción. Las deleciones intragénicas menores a $30 \mathrm{~kb}$ no se pueden identificar mediante microarreglos y deberá realizarse secuenciación. ${ }^{1,2,4,8}$ En el presente caso no se realizó el estudio de microarreglos de primera intención, se inició con el cariotipo con lo cual fue posible establecer la alteración estructural y posteriormente confirmar el diagnóstico mediante las diferentes metodologías antes mencionas.

\section{Diagnóstico diferencial}

La mayoría de los diagnósticos diferenciales son desórdenes del neurodesarrollo con datos clínicos que se sobreponen con el SPMD. Entre los principales síndromes se encuentran: PraderWilli, por la hipotonía al nacimiento; Angelman, debido al retardo global del desarrollo, ausencia del lenguaje y comportamiento autista; síndrome de X-frágil por ser la causa más frecuente de retardo en el neuro-desarrollo en varones y síndrome de Sotos, principalmente. Es importante descartar en los desórdenes con espectro autista una entidad sindromática como el SPMD y distinguirlo de entidades idiopáticas, al igual que en pacientes previamente diagnosticados con parálisis cerebral. ${ }^{1,2,4}$

\section{Diagnóstico prenatal}

No se han descrito alteraciones específicas en el estudio ultrasonográfico. El cariotipo y los estudios moleculares se pueden realizar tanto en vellosidades coriales como en el líquido amniótico, los cuales están indicados en casos con antecedentes familiares o cuando los padres son portadores de re-arreglos cromosómicos balanceados que involucren al cromosoma afectado. En ocasiones puede ser un hallazgo cuando el estudio se ha realizado por otros motivos como la edad materna avanzada.

\section{Asesoramiento y bases genéticas}

Si bien el 80\% de los pacientes con SPMD son casos esporádicos o de novo y el riesgo de recurrencia es prácticamente nulo, está indicado realizar estudios en los progenitores con un hijo con deleción 22q13.3 debido a la posibilidad de un mosaico o de un re-arreglo estructural en alguno de ellos. En 20\% de los casos la deleción se debe a un desequilibrio estructural, como una translocación o la formación de un anillo y, de éstos, $50 \%$ se deben a un re-arreglo balanceado en alguno de los progenitores, lo cual eleva el riesgo de recurrencia en un siguiente embarazo. Es importante considerar en los casos aparentemente de novo la posibilidad de mosaico germinal en alguno de los padres, lo que incrementaría el riesgo de recurrencia en un siguiente embarazo aproximadamente en un $2 \%$. $^{1,8}$

El tamaño de la deleción varía de 130 kb hasta $9 \mathrm{Mb} ;{ }^{2,8,9}$ sin embargo, la región crítica del SPMD se ha ido redefiniendo a un área aproximadamente de $100 \mathrm{~kb}$, que contiene tres genes: $A C R$, $S H A N K 3$ y RABL2B. El gen $A C R$ codifica para una proteína vinculada con la espermatogénesis y es muy poco probable que su pérdida esté 
relacionada con el SPMD. El gen $R A B L 2 B$ codifica para una proteína $G$, que regula el tráfico vesicular intracelular, pero la expresión del gen $R A B L 2 A$ localizado en el cromosoma 2, parece compensar la pérdida del gen $R A B L 2 B$. De tal manera que el gen $S H A N K 3$, también conocido como ProsSAP2, es el gen relacionado con la mayor parte del fenotipo observado en el SPMD. Esto se ha evidenciado en diversos reportes de caso que han sido resultado de pequeñas deleciones o mutaciones que sólo involucran a SHANK3 con SPMD. ${ }^{4}$ De modo que, independientemente del tamaño de la deleción, el énfasis en la génesis del síndrome se ha puesto en la pérdida de $S H A N K 3$, un gen que consta de 22 exones, con 5 islas CpG (citosina-guanina) y se sabe es regulado epigenéticamente. Se expresa abundantemente en el corazón y de forma moderada en el bazo y en el cerebro. Codifica para una proteína multidominio, que en el cerebro se ubica fundamentalmente en la densidad postsináptica de las neuronas glutaminérgicas excitatorias. Interviene tanto en la función como en plasticidad neuronal e interactúa con diversos receptores como N-metil-aspartato (NMDA$\mathrm{R})$, el receptor glutaminérgico metabotrópico (mGLURs) y el receptor a-amino-3-hidroxi5-metil-4-ácido-propiónico (AMPA-R). Además parece desempeñar un papel importante en el aprendizaje y en la memoria; también hay evidencia de que interviene en la transducción de señales en las células del sistema inmunológico. ${ }^{2,9-11}$ En algunos pacientes se ha descrito la deleción de un gen IB2, localizado a $70 \mathrm{~kb}$ de SHANK3 y puede estar asociado al SPMD pues parece contribuir a la estabilidad sináptica y a la transmisión neuronal. ${ }^{12}$

\section{Correlación fenotipo-genotipo}

En una cohorte de 201 pacientes, la más grande realizada hasta el momento y en estudios más pequeños, han observado cierta asociación entre el tamaño de la deleción y el fenotipo, lo que indica el papel de otros genes, así como efectos de posición y de elementos regulatorios en el SPMD. En general las deleciones de mayor tamaño se relacionan con una mayor severidad en el retraso psicomotor, ausencia de lenguaje, macrocefalia, dismorfias faciales y manos grandes. Las asociaciones más consistentes y de mayor significancia estadística son: la macrocefalia que se asocia a deleciones mayores $5 \mathrm{Mb}$ y el desarrollo del lenguaje que es inversamente proporcional al tamaño de la deleción; es decir, aquellos pacientes con deleciones de 9.2 Mb se asocian a ausencia del lenguaje y aquellos con deleciones de $4.5 \mathrm{Mb}$ con lenguaje funcional. ${ }^{2,6,7,9}$ Esto ha dado pie a investigar el rol de otros genes como MPPED1, CYB5R3 en 22q13.2 y FBLN1, NUP50, $P A R V B, T R M U, W N T 7 B$ y algunos microRNA's en $22 q 13.31 .{ }^{6}$ Uno de estos vínculos se ha descubierto en el caso de la macrocefalia debido a que las deleciones mayores a $5 \mathrm{Mb}$ incluyen al gen $W N T 7 B$, que interactúa con la proteína GPC3 involucrada en el síndrome con macrocefalia Simpson-Golabi-Behmel. Aún se requieren más estudios prospectivos que incluyan como variantes la edad y el tamaño de la deleción para delinear mejor el espectro fenotípico del SPMD. ${ }^{6}$

En nuestro paciente, a pesar de que la deleción es de $4.3 \mathrm{Mb}$, observamos la pérdida total de lenguaje que no coincide con lo reportado pero, efectivamente, no hay macrocefalia.

\section{SEGUIMIENTO}

Los pacientes con SPMD requieren la asistencia de un grupo multidisciplinario de especialistas, de acuerdo a las manifestaciones clínicas previamente descritas. ${ }^{2}$ Ahora se sabe que la expresividad de la enfermedad depende de la edad, por lo que el seguimiento de las diferentes manifestaciones descritas tendrán que ser de forma continua a lo largo de la vida, ya que el riesgo de espectro autista, crisis convulsivas, linfedema y celulitis incrementa con la edad.6,7 


\section{AVANCES EN LA INVESTIGACIÓN DE NUEVOS TRATAMIENTOS}

Hasta el momento se han publicado tres estudios clínicos que analizan la respuesta al tratamiento farmacológico y algunos otros están en desarroIlo. El primero es la administración de insulina intranasal debido que se ha observado mejoría de la memoria en pacientes con Alzheimer y se considera puede lograr mejoría de algunos síntomas neurológicos en pacientes con deleción $22 q 13.3$.

El estudio de Schmidt y sus colaboradores mostró mejoría a corto y largo plazo en las habilidades motoras finas y gruesas, así como en funciones cognitivas en pacientes con SPMD. El mecanismo por el cual parece actuar la insulina intranasal es la interacción con el receptor llamado IRSp53 (insulin receptor kinase substrate) el que a su vez interactúa con SHANK3 y su activación juega un papel en la organización sináptica. Por otro lado, incrementa la expresión de la proteína PSD59, la cual puede parcialmente contrarrestar la deficiencia de SHANK3. A pesar de que en este estudio no se observaron efectos adversos, es indispensable valorar a los pacientes a largo plazo. ${ }^{2,4}$ El segundo estudio es mediante el uso de risperidona en dosis bajas que actúa a nivel de los receptores glutaminérgicos. ${ }^{2,4} \mathrm{El}$ tercer estudio involucra el uso del factor de crecimiento parecido a la insulina tipo 1 (IGF-1) debido a que los factores de crecimiento juegan un papel importante en el desarrollo del sistema nervioso central. IGF-1 es un polipéptido pequeño capaz de atravesar la barrera hematoencefálica y promueve la maduración neuronal y la formación de sinapsis. El estudio clínico para la administración de IGF-1 en una primera fase con 9 pacientes entre 5 y 15 años ha mostrado resultados preliminares con adecuada tolerabilidad y ningún efecto adverso grave, actualmente está en fase 2; este mismo estudio ha mostrado resultados positivos en pacientes con síndrome de Rett. ${ }^{4}$

\section{PRONÓSTICO}

Los pacientes no suelen tener alguna alteración que ponga en riesgo la vida; no obstante, se desconoce la evolución de estos pacientes hasta la edad adulta. Es una entidad que requiere atención durante toda la vida y se necesita supervisión de un cuidador. ${ }^{2}$

\section{CONCLUSIONES}

El SPMD es una entidad con mayor frecuencia de la esperada, está sub-diagnosticada y se espera que mediante su difusión y la tecnología diagnóstica actual los pacientes y sus familiares se beneficien de un diagnóstico más temprano, oportuno y de un mejor manejo.

El caso presentado ha tenido una evolución altamente sugestiva de SPMD desde el nacimiento y, sin embargo, el diagnóstico se realizó hasta los cuatro años. Todo parece indicar que el espectro fenotípico es muy amplio por lo que, en la medida en la que el estudio de microarreglos se introduzca como una práctica clínica habitual para pacientes con retardo del desarrollo, con o sin dismorfias, habrá un mayor número de pacientes diagnosticados. Es importante recalcar que es fundamental realizarlo en conjunto con el cariotipo, ya que brindan información complementaria para el establecimiento de la etiología.

La deficiencia del gen SHANK3 representa una de las causas monogenénicas más frecuentes de autismo y de retardo del desarrollo, algunas referencias reportan hasta 5 y $1 \%$, respectivamente, por lo que es importante considerarlo dentro los posibles diagnósticos diferenciales en pacientes con estas dos manifestaciones. ${ }^{10}$ Recientemente se ha descubierto que también desempeña un papel importante en la génesis de diversas alteraciones neuropsiquiátricas como la esquizofrenia, por lo que el estudio de la función y regulación de SHANK3 pudiera tener mayor alcance. ${ }^{11}$ 
Hernández-Gómez M et al. Síndrome de Phelan-McDermid

\section{REFERENCIAS}

1. Phelan MC. Deletion 22q13. 3 syndrome. Orphanet journal of rare diseases. 2008;3(1):1. DOI: 10.1186/17501172-3-14

2. Phelan $\mathrm{K}, \mathrm{McD}$ ermid $\mathrm{H}$. The 22q13. 3 deletion syndrome (Phelan-McDermid syndrome). Molecular syndromology. 2011;2(3-5):186-201.

3. Canonero I, Montes C, Sturich A, Boterón M, Asinari M, Cuestas E, et al. Síndrome de Phelan McDermid: descripción de cinco pacientes e informe del primer caso descripto en gemelas siamesas. Archivos argentinos de pediatría. 2012;110(3):e50-e4.

4. Costales JL, Kolevzon A. Phelan-McDermid Syndrome and SHANK3: Implications for Treatment. Neurotherapeutics. 2015;12(3):620-30.

5. Miller DT, Adam MP, Aradhya S, Biesecker LG, Brothman AR, Carter NP, et al. Consensus statement: chromosomal microarray is a first-tier clinical diagnostic test for individuals with developmental disabilities or congenital anomalies. The American Journal of Human Genetics. 2010;86(5):749-64.

6. Sarasua SM, Boccuto L, Sharp JL, Dwivedi A, Chen C-F, Rollins JD, et al. Clinical and genomic evaluation of 201 patients with Phelan-McDermid syndrome. Human genetics. 2014;133(7):847-59.
7. Oberman LM, Boccuto L, Cascio L, Sarasua S, Kaufmann WE. Autism spectrum disorder in Phelan-McDermid syndrome: initial characterization and genotype-phenotype correlations. Orphanet journal of rare diseases. 2015;10(1):1. DOI: 10.1186/s13023-015-0323-9

8. Kolevzon A, Angarita B, Bush L, Wang AT, Frank Y, Yang A, et al. Phelan-McDermid syndrome: a review of the literature and practice parameters for medical assessment and monitoring. Journal of neurodevelopmental disorders. 2014;6(1):1. doi: 10.1186/1866-1955-6-39

9. Dhar S, Del Gaudio D, German J, Peters S, Ou Z, Bader P, et al. 22q13. 3 deletion syndrome: clinical and molecular analysis using array $\mathrm{CGH}$. American Journal of Medical Genetics Part A. 2010;152(3):573-81.

10. Soorya L, Kolevzon A, Zweifach J, Lim T, Dobry Y, Schwartz $L$, et al. Prospective investigation of autism and genotypephenotype correlations in 22q13 deletion syndrome and SHANK3 deficiency. Molecular autism. 2013;4(1):1. doi: 10.1186/2040-2392-4-18

11. Uchino S, Waga C. SHANK3 as an autism spectrum disorderassociated gene. Brain and Development. 2013;35(2):10610.

12. Lei D, Li S, Banerjee S, Zhang H, Li C, Hou S, et al. Clinical and genomic evaluation of a Chinese patient with a novel deletion associated with Phelan-McDermid syndrome. Oncotarget. 2016;(49):80327-80335. 